\title{
Applications of quantum calculus on finite intervals to impulsive difference inclusions
}

Sotiris K Ntouyas ${ }^{1}$ and Jessada Tariboon ${ }^{2,3^{*}}$

${ }^{*}$ Correspondence:

jessadat@kmutnb.ac.th

${ }^{2}$ Department of Mathematics,

Faculty of Applied Science,

Nonlinear Dynamic Analysis Research Center, King Mongkut's

University of Technology North

Bangkok, Bangkok, 10800, Thailand

${ }^{3}$ Centre of Excellence in

Mathematics, CHE, Si Ayutthaya Rd.

Bangkok, 10400, Thailand

Full list of author information is

available at the end of the article

\begin{abstract}
Recently Tariboon and Ntouyas (Adv. Differ. Equ. 2013:282, 2013) introduced the notions of $q_{k}$-derivative and $q_{k}$-integral of a function on finite intervals. As applications existence and uniqueness results for initial value problems for first- and second-order impulsive $q_{k}$-difference equations was proved. In this paper, continuing the study of Tariboon and Ntouyas (Adv. Differ. Equ. 2013:282, 2013), we apply the quantum calculus to initial value problems for impulsive first- and second-order $q_{k}$-difference inclusions. We establish new existence results, when the right hand side is convex valued, by using the nonlinear alternative of Leray-Schauder type. Some illustrative examples are also presented.

MSC: 34A60; 26A33; 39A13; 34A37
\end{abstract}

Keywords: $q_{k}$-derivative; $q_{k}$-integral; impulsive $q$-difference inclusion

\section{Introduction and preliminaries}

In [1] the notions of $q_{k}$-derivative and $q_{k}$-integral of a function $f: J_{k}:=\left[t_{k}, t_{k+1}\right] \rightarrow \mathbb{R}$, have been introduced and their basic properties was proved. As applications, existence and uniqueness results for initial value problems for first- and second-order impulsive $q_{k}$ difference equations was proved.

We recall the notions of $q_{k}$-derivative and $q_{k}$-integral on finite intervals. For a fixed $k \in$ $\mathbb{N} \cup\{0\}$ let $J_{k}:=\left[t_{k}, t_{k+1}\right] \subset \mathbb{R}$ be an interval and $0<q_{k}<1$ be a constant. We define $q_{k}$ derivative of a function $f: J_{k} \rightarrow \mathbb{R}$ at a point $t \in J_{k}$ as follows.

Definition 1.1 Assume $f: J_{k} \rightarrow \mathbb{R}$ is a continuous function and let $t \in J_{k}$. Then the expression

$$
D_{q_{k}} f(t)=\frac{f(t)-f\left(q_{k} t+\left(1-q_{k}\right) t_{k}\right)}{\left(1-q_{k}\right)\left(t-t_{k}\right)}, \quad t \neq t_{k}, \quad D_{q_{k}} f\left(t_{k}\right)=\lim _{t \rightarrow t_{k}} D_{q_{k}} f(t)
$$

is called the $q_{k}$-derivative of function $f$ at $t$.

We say that $f$ is $q_{k}$-differentiable on $J_{k}$ provided $D_{q_{k}} f(t)$ exists for all $t \in J_{k}$. Note that if $t_{k}=0$ and $q_{k}=q$ in (1.1), then $D_{q_{k}} f=D_{q} f$, where $D_{q}$ is the well-known $q$-derivative of the function $f(t)$ defined by

$$
D_{q} f(t)=\frac{f(t)-f(q t)}{(1-q) t} .
$$

O2014 Ntouyas and Tariboon; licensee Springer. This is an Open Access article distributed under the terms of the Creative Commons Attribution License (http://creativecommons.org/licenses/by/2.0), which permits unrestricted use, distribution, and reproduction in any medium, provided the original work is properly cited. 
In addition, we should define the higher $q_{k}$-derivative of functions.

Definition 1.2 Let $f: J_{k} \rightarrow \mathbb{R}$ is a continuous function, we call the second-order $q_{k}$ derivative $D_{q_{k}}^{2} f$ provided $D_{q_{k}} f$ is $q_{k}$-differentiable on $J_{k}$ with $D_{q_{k}}^{2} f=D_{q_{k}}\left(D_{q_{k}} f\right): J_{k} \rightarrow \mathbb{R}$. Similarly, we define higher order $q_{k}$-derivative $D_{q_{k}}^{n}: J_{k} \rightarrow \mathbb{R}$.

The properties of $q_{k}$-derivative are discussed in [1].

Definition 1.3 Assume $f: J_{k} \rightarrow \mathbb{R}$ is a continuous function. Then the $q_{k}$-integral is defined by

$$
\int_{t_{k}}^{t} f(s) d_{q_{k}} s=\left(1-q_{k}\right)\left(t-t_{k}\right) \sum_{n=0}^{\infty} q_{k}^{n} f\left(q_{k}^{n} t+\left(1-q_{k}^{n}\right) t_{k}\right)
$$

for $t \in J_{k}$. Moreover, if $a \in\left(t_{k}, t\right)$ then the definite $q_{k}$-integral is defined by

$$
\begin{aligned}
\int_{a}^{t} f(s) d_{q_{k}} s= & \int_{t_{k}}^{t} f(s) d_{q_{k}} s-\int_{t_{k}}^{a} f(s) d_{q_{k}} s=\left(1-q_{k}\right)\left(t-t_{k}\right) \sum_{n=0}^{\infty} q_{k}^{n} f\left(q_{k}^{n} t+\left(1-q_{k}^{n}\right) t_{k}\right) \\
& -\left(1-q_{k}\right)\left(a-t_{k}\right) \sum_{n=0}^{\infty} q_{k}^{n} f\left(q_{k}^{n} a+\left(1-q_{k}^{n}\right) t_{k}\right) .
\end{aligned}
$$

Note that if $t_{k}=0$ and $q_{k}=q$, then (1.3) reduces to $q$-integral of a function $f(t)$, defined by $\int_{0}^{t} f(s) d_{q} s=(1-q) t \sum_{n=0}^{\infty} q^{n} f\left(q^{n} t\right)$ for $t \in[0, \infty)$.

The book by Kac and Cheung [2] covers many of the fundamental aspects of the quantum calculus. In recent years, the topic of $q$-calculus has attracted the attention of several researchers and a variety of new results can be found in the papers [3-15] and the references cited therein.

Impulsive differential equations, that is, differential equations involving the impulse effect, appear as a natural description of observed evolution phenomena of several real world problems. For some monographs on the impulsive differential equations we refer to $[16-18]$.

Here, we remark that the classical $q$-calculus cannot be considered in problems with impulses as the definition of $q$-derivative fails to work when there are impulse points $t_{k} \in(q t, t)$ for some $k \in \mathbb{N}$. On the other hand, this situation does not arise for impulsive problems on a $q$-time scale as the points $t$ and $q t=\rho(t)$ are consecutive points, where $\rho: \mathbb{T} \rightarrow \mathbb{T}$ is the backward jump operator; see [19]. In [1], quantum calculus on finite intervals, the points $t$ and $q_{k} t+\left(1-q_{k}\right) t_{k}$ are considered only in an interval $\left[t_{k}, t_{k+1}\right]$. Therefore, the problems with impulses at fixed times can be considered in the framework of $q_{k}$-calculus.

In this paper, continuing the study of [1], we apply $q_{k}$-calculus to establish existence results for initial value problems for impulsive first- and second-order $q_{k}$-difference inclusions. In Section 3, we consider the following initial value problem for the first-order $q_{k}$-difference inclusion:

$$
\begin{aligned}
& D_{q_{k}} x(t) \in F(t, x(t)), \quad t \in J:=[0, T], t \neq t_{k}, \\
& \Delta x\left(t_{k}\right)=I_{k}\left(x\left(t_{k}\right)\right), \quad k=1,2, \ldots, m, \\
& x(0)=x_{0},
\end{aligned}
$$


where $x_{0} \in \mathbb{R}, 0=t_{0}<t_{1}<t_{2}<\cdots<t_{k}<\cdots<t_{m}<t_{m+1}=T, f:[0, T] \times \mathbb{R} \rightarrow \mathcal{P}(\mathbb{R})$ is a multivalued function, $\mathcal{P}(\mathbb{R})$ is the family of all nonempty subjects of $\mathbb{R}, I_{k} \in C(\mathbb{R}, \mathbb{R})$, $\Delta x\left(t_{k}\right)=x\left(t_{k}^{+}\right)-x\left(t_{k}\right), k=1,2, \ldots, m$ and $0<q_{k}<1$ for $k=0,1,2, \ldots, m$.

In Section 4, we study the existence of solutions for the following initial value problem for second-order impulsive $q_{k}$-difference inclusion:

$$
\begin{aligned}
& D_{q_{k}}^{2} x(t) \in F(t, x(t)), \quad t \in J, t \neq t_{k}, \\
& \Delta x\left(t_{k}\right)=I_{k}\left(x\left(t_{k}\right)\right), \quad k=1,2, \ldots, m, \\
& D_{q_{k}} x\left(t_{k}^{+}\right)-D_{q_{k-1}} x\left(t_{k}\right)=I_{k}^{*}\left(x\left(t_{k}\right)\right), \quad k=1,2, \ldots, m, \\
& x(0)=\alpha, \quad D_{q_{0}} x(0)=\beta,
\end{aligned}
$$

where $\alpha, \beta \in \mathbb{R}$ and $I_{k}, I_{k}^{*} \in C(\mathbb{R}, \mathbb{R})$.

We establish new existence results, when the right hand side is convex valued by using the nonlinear alternative of Leray-Schauder type.

The paper is organized as follows. In Section 2, we recall some preliminary facts that we need in the sequel. In Section 3 we establish the existence result for first-order $q_{k}$ difference inclusions, while the existence result for second-order $q_{k}$-difference inclusions is presented in Section 4. Some illustrative examples are also presented.

\section{Preliminaries}

In this section we recall some basic concepts of multivalued analysis [20,21].

For a normed space $(X,\|\cdot\|)$, let $\mathcal{P}_{c l}(X)=\{Y \in \mathcal{P}(X): Y$ is closed $\}, \mathcal{P}_{c p}(X)=\{Y \in \mathcal{P}(X)$ : $Y$ is compact $\}$, and $\mathcal{P}_{c p, c}(X)=\{Y \in \mathcal{P}(X): Y$ is compact and convex $\}$.

A multivalued map $G: X \rightarrow \mathcal{P}(X)$ is convex (closed) valued if $G(x)$ is convex (closed) for all $x \in X$; is bounded on bounded sets if $G(\mathbb{B})=\bigcup_{x \in \mathbb{B}} G(x)$ is bounded in $X$ for all $\mathbb{B} \in \mathcal{P}_{b}(X)$ (i.e. $\sup _{x \in \mathbb{B}}\{\sup \{|y|: y \in G(x)\}\}<\infty$ ); is called upper semicontinuous (u.s.c.) on $X$ if for each $x_{0} \in X$, the set $G\left(x_{0}\right)$ is a nonempty closed subset of $X$, and if for each open set $N$ of $X$ containing $G\left(x_{0}\right)$, there exists an open neighborhood $\mathcal{N}_{0}$ of $x_{0}$ such that $G\left(\mathcal{N}_{0}\right) \subseteq N$; is said to be completely continuous if $G(\mathbb{B})$ is relatively compact for every $\mathbb{B} \in \mathcal{P}_{b}(X)$.

In the sequel, we denote by $\mathcal{C}=C([0, T], \mathbb{R})$ the space of all continuous functions from $[0, T] \rightarrow \mathbb{R}$ with norm $\|x\|=\sup \{|x(t)|: t \in[0, T]\}$. By $L^{1}([0, T], \mathbb{R})$ we denote the space of all functions $f$ defined on $[0, T]$ such that $\|x\|_{L^{1}}=\int_{0}^{T}|x(t)| d t<\infty$.

For each $y \in \mathcal{C}$, define the set of selections of $F$ by

$$
S_{F, y}:=\{v \in \mathcal{C}: v(t) \in F(t, y(t)) \text { on }[0, T]\} .
$$

Definition 2.1 A multivalued map $F: J \times \mathbb{R} \rightarrow \mathcal{P}(\mathbb{R})$ is said to be Carathéodory (in the sense of $q_{k}$-calculus) if $x \longmapsto F(t, x)$ is upper semicontinuous on $J$. Further a Carathéodory function $F$ is called $L^{1}$-Carathéodory if there exists $\varphi_{\alpha} \in L^{1}\left(J, \mathbb{R}^{+}\right)$such that $\|F(t, x)\|=$ $\sup \{|v|: v \in F(t, x)\} \leq \varphi_{\alpha}(t)$ for all $\|x\| \leq \alpha$ on $J$ for each $\alpha>0$.

We recall the well-known nonlinear alternative of Leray-Schauder for multivalued maps and a useful result regarding closed graphs.

Lemma 2.2 (Nonlinear alternative for Kakutani maps) [22] Let E be a Banach space, $C$ a closed convex subset of $E, U$ an open subset of $C$ and $0 \in U$. Suppose that $F: \bar{U} \rightarrow \mathcal{P}_{c p, c}(C)$ 
is a upper semicontinuous compact map. Then either

(i) F has a fixed point in $\bar{U}$, or

(ii) there is $a u \in \partial U$ and $\lambda \in(0,1)$ with $u \in \lambda F(u)$.

Lemma $2.3([23,24])$ Let $X$ be a Banach space. Let $F: J \times \mathbb{R} \rightarrow \mathcal{P}_{c p, c}(X)$ be an $L^{1}$ Carathéodory multivalued map and let $\Theta$ be a linear continuous mapping from $L^{1}(J, \mathbb{R})$ to $C(J, \mathbb{R})$. Then the operator

$$
\Theta \circ S_{F}: C(J, \mathbb{R}) \rightarrow \mathcal{P}_{c p, c}(C(J, \mathbb{R})), \quad x \mapsto\left(\Theta \circ S_{F}\right)(x)=\Theta\left(S_{F, x}\right)
$$

is a closed graph operator in $C(J, \mathbb{R}) \times C(J, \mathbb{R})$.

Let $J=[0, T], J_{0}=\left[t_{0}, t_{1}\right], J_{k}=\left(t_{k}, t_{k+1}\right]$ for $k=1,2, \ldots, m$. Let $P C(J, \mathbb{R})=\{x: J \rightarrow \mathbb{R}: x(t)$ is continuous everywhere except for some $t_{k}$ at which $x\left(t_{k}^{+}\right)$and $x\left(t_{k}^{-}\right)$exist and $x\left(t_{k}^{-}\right)=x\left(t_{k}\right)$, $k=1,2, \ldots, m\} . P C(J, \mathbb{R})$ is a Banach space with the norms $\|x\|_{P C}=\sup \{|x(t)| ; t \in J\}$.

\section{First-order impulsive $q_{k}$-difference inclusions}

In this section, we study the existence of solutions for the first-order impulsive $q_{k}$ difference inclusion (1.4).

The following lemma was proved in [1].

Lemma 3.1 If $y \in P C(J, \mathbb{R})$, then for any $t \in J_{k}, k=0,1,2, \ldots, m$, the solution of the problem

$$
\begin{aligned}
& D_{q_{k}} x(t)=y(t), \quad t \in J, t \neq t_{k}, \\
& \Delta x\left(t_{k}\right)=I_{k}\left(x\left(t_{k}\right)\right), \quad k=1,2, \ldots, m, \\
& x(0)=x_{0}
\end{aligned}
$$

is given by

$$
x(t)=x_{0}+\sum_{0<t_{k}<t} \int_{t_{k-1}}^{t_{k}} y(s) d_{q_{k-1}} s+\sum_{0<t_{k}<t} I_{k}\left(x\left(t_{k}\right)\right)+\int_{t_{k}}^{t} y(s) d_{q_{k}} s,
$$

with $\sum_{0<0}(\cdot)=0$.

Before studying the boundary value problem (1.4) let us begin by defining its solution.

Definition 3.2 A function $x \in P C(J, \mathbb{R})$ is said to be a solution of (1.4) if $x(0)=x_{0}, \Delta x\left(t_{k}\right)=$ $I_{k}\left(x\left(t_{k}\right)\right), k=1,2, \ldots, m$, and there exists $f \in L^{1}(J, \mathbb{R})$ such that $f(t) \in F(t, x(t))$ on $J$ and

$$
x(t)=x_{0}+\sum_{0<t_{k}<t} \int_{t_{k-1}}^{t_{k}} f(s) d_{q_{k-1}} s+\sum_{0<t_{k}<t} I_{k}\left(x\left(t_{k}\right)\right)+\int_{t_{k}}^{t} f(s) d_{q_{k}} s .
$$

Theorem 3.3 Assume that:

$\left(\mathrm{H}_{1}\right) \quad F: J \times \mathbb{R} \rightarrow \mathcal{P}(\mathbb{R})$ is Carathéodory and has nonempty compact and convex values; 
$\left(\mathrm{H}_{2}\right)$ there exist a continuous nondecreasing function $\psi:[0, \infty) \rightarrow(0, \infty)$ and a function $p \in C\left(J, \mathbb{R}^{+}\right)$such that

$$
\|F(t, x)\|_{\mathcal{P}}:=\sup \{|y|: y \in F(t, x)\} \leq p(t) \psi(\|x\|) \quad \text { for each }(t, x) \in J \times \mathbb{R}
$$

$\left(\mathrm{H}_{3}\right)$ there exist constants $c_{k}$ such that $\left|I_{k}(y)\right| \leq c_{k}, k=1,2, \ldots$, m for each $y \in \mathbb{R}$;

$\left(\mathrm{H}_{4}\right)$ there exists a constant $M>0$ such that

$$
\frac{M}{\left|x_{0}\right|+T \psi(M)\|p\|+\sum_{k=1}^{m} c_{k}}>1 \text {. }
$$

Then the initial value problem (1.4) has at least one solution on J.

Proof Define the operator $\mathcal{H}: P C(J, \mathbb{R}) \rightarrow \mathcal{P}(P C(J, \mathbb{R}))$ by

$$
\mathcal{H}(x)=h \in P C(J, \mathbb{R}): h(t)=x_{0}+\sum_{0<t_{k}<t} \int_{t_{k-1}}^{t_{k}} f(s) d_{q_{k-1}} s+\sum_{0<t_{k}<t} I_{k}\left(x\left(t_{k}\right)\right)+\int_{t_{k}}^{t} f(s) d_{q_{k}} s,
$$

for $f \in S_{F, x}$.

We will show that $\mathcal{H}$ satisfies the assumptions of the nonlinear alternative of LeraySchauder type. The proof consists of several steps. As a first step, we show that $\mathcal{H}$ is convex for each $x \in P C(J, \mathbb{R})$. This step is obvious since $S_{F, x}$ is convex ( $F$ has convex values), and therefore we omit the proof.

In the second step, we show that $\mathcal{H}$ maps bounded sets (balls) into bounded sets in $P C(J, \mathbb{R})$. For a positive number $\rho$, let $B_{\rho}=\{x \in C(J, \mathbb{R}):\|x\| \leq \rho\}$ be a bounded ball in $C(J, \mathbb{R})$. Then, for each $h \in \mathcal{H}(x), x \in B_{\rho}$, there exists $f \in S_{F, x}$ such that

$$
h(t)=x_{0}+\sum_{0<t_{k}<t} \int_{t_{k-1}}^{t_{k}} f(s) d_{q_{k-1}} s+\sum_{0<t_{k}<t} I_{k}\left(x\left(t_{k}\right)\right)+\int_{t_{k}}^{t} f(s) d_{q_{k}} s .
$$

Then for $t \in J$ we have

$$
\begin{aligned}
|h(t)| & \leq\left|x_{0}\right|+\sum_{0<t_{k}<t} \int_{t_{k-1}}^{t_{k}}|f(s)| d_{q_{k-1}} s+\sum_{0<t_{k}<t}\left|I_{k}\left(x\left(t_{k}\right)\right)\right|+\int_{t_{k}}^{t}|f(s)| d_{q_{k}} s \\
& \leq\left|x_{0}\right|+\sum_{0<t_{k}<t} \int_{t_{k-1}}^{t_{k}} p(s) \psi(\|x\|) d_{q_{k-1}} s+\sum_{k=1}^{m} c_{k}+\int_{t_{k}}^{t} p(s) \psi(\|x\|) d_{q_{k}} s \\
& \leq\left|x_{0}\right|+\psi(\|x\|) \sum_{0<t_{k}<t} \int_{t_{k-1}}^{t_{k}} p(s) d_{q_{k-1}} s+\sum_{k=1}^{m} c_{k}+\psi(\|x\|) \int_{t_{k}}^{t} p(s) d_{q_{k}} s \\
& \leq\left|x_{0}\right|+T \psi(\|x\|)\|p\|+\sum_{k=1}^{m} c_{k} .
\end{aligned}
$$

Consequently,

$$
\|h\| \leq\left|x_{0}\right|+T \psi(\rho)\|p\|+\sum_{k=1}^{m} c_{k} .
$$


Now we show that $\mathcal{H}$ maps bounded sets into equicontinuous sets of $P C(J, \mathbb{R})$. Let $\tau_{1}, \tau_{2} \in J, \tau_{1}<\tau_{2}$ with $\tau_{1} \in J_{v}, \tau_{2} \in J_{u}, v \leq u$ for some $u, v \in\{0,1,2, \ldots, m\}$ and $x \in B_{\rho}$. For each $h \in \mathcal{H}(x)$, we obtain

$$
\begin{aligned}
\left|h\left(\tau_{2}\right)-h\left(\tau_{1}\right)\right| \leq & \left|\int_{t_{u}}^{\tau_{2}} f(s) d_{q_{k}} s-\int_{t_{v}}^{\tau_{1}} f(s) d_{q_{k}} s\right|+\left|\sum_{\tau_{1}<t_{k}<\tau_{2}} I_{k}\left(x\left(t_{k}\right)\right)\right| \\
& +\left|\sum_{\tau_{1}<t_{k}<\tau_{2}} \int_{t_{k-1}}^{t_{k}} f(s) d_{q_{k-1}} s\right| \\
\leq & \left|\int_{t_{u}}^{\tau_{2}} f(s) d_{q_{k}} s-\int_{t_{v}}^{\tau_{1}} f(s) d_{q_{k}} s\right|+\sum_{\tau_{1}<t_{k}<\tau_{2}}\left|I_{k}\left(x\left(t_{k}\right)\right)\right| \\
& +\sum_{\tau_{1}<t_{k}<\tau_{2}} \int_{t_{k-1}}^{t_{k}}|f(s)| d_{q_{k-1}} s .
\end{aligned}
$$

Obviously the right hand side of the above inequality tends to zero independently of $x \in$ $B_{\rho}$ as $\tau_{2}-\tau_{1} \rightarrow 0$. Therefore it follows by the Arzelá-Ascoli theorem that $\mathcal{H}: P C(J, \mathbb{R}) \rightarrow$ $\mathcal{P}(P C(J, \mathbb{R}))$ is completely continuous.

Since $\mathcal{H}$ is completely continuous, in order to prove that it is upper semicontinuous it is enough to prove that it has a closed graph. Thus, in our next step, we show that $\mathcal{H}$ has a closed graph. Let $x_{n} \rightarrow x_{*}, h_{n} \in \mathcal{H}\left(x_{n}\right)$ and $h_{n} \rightarrow h_{*}$. Then we need to show that $h_{*} \in \mathcal{H}\left(x_{*}\right)$. Associated with $h_{n} \in \mathcal{H}\left(x_{n}\right)$, there exists $f_{n} \in S_{F, x_{n}}$ such that, for each $t \in J$,

$$
h_{n}(t)=x_{0}+\sum_{0<t_{k}<t} \int_{t_{k-1}}^{t_{k}} f_{n}(s) d_{q_{k-1}} s+\sum_{0<t_{k}<t} I_{k}\left(x_{n}\left(t_{k}\right)\right)+\int_{t_{k}}^{t} f_{n}(s) d_{q_{k}} s .
$$

Thus it suffices to show that there exists $f_{*} \in S_{F, x_{*}}$ such that, for each $t \in J$,

$$
h_{*}(t)=x_{0}+\sum_{0<t_{k}<t} \int_{t_{k-1}}^{t_{k}} f_{*}(s) d_{q_{k-1}} s+\sum_{0<t_{k}<t} I_{k}\left(x_{*}\left(t_{k}\right)\right)+\int_{t_{k}}^{t} f_{*}(s) d_{q_{k}} s .
$$

Let us consider the linear operator $\Theta: L^{1}(J, \mathbb{R}) \rightarrow P C(J, \mathbb{R})$ given by

$$
f \mapsto \Theta(f)(t)=x_{0}+\sum_{0<t_{k}<t} \int_{t_{k-1}}^{t_{k}} f(s) d_{q_{k-1}} s+\sum_{0<t_{k}<t} I_{k}\left(x\left(t_{k}\right)\right)+\int_{t_{k}}^{t} f(s) d_{q_{k}} s .
$$

Observe that

$$
\begin{aligned}
\left\|h_{n}(t)-h_{*}(t)\right\|= & \| \sum_{0<t_{k}<t} \int_{t_{k-1}}^{t_{k}}\left(f_{n}(u)-f_{*}(u)\right) d_{q_{k-1}} s+\sum_{0<t_{k}<t}\left|I_{k}\left(x_{n}\left(t_{k}\right)\right)-I_{k}\left(x_{*}\left(t_{k}\right)\right)\right| \\
& +\int_{t_{k}}^{t}\left(f_{n}(u)-f_{*}(u)\right) d_{q_{k}} s \| \rightarrow 0,
\end{aligned}
$$

as $n \rightarrow \infty$. 
Thus, it follows by Lemma 2.3 that $\Theta \circ S_{F}$ is a closed graph operator. Further, we have $h_{n}(t) \in \Theta\left(S_{F, x_{n}}\right)$. Since $x_{n} \rightarrow x_{*}$, therefore, we have

$$
h_{*}(t)=x_{0}+\sum_{0<t_{k}<t} \int_{t_{k-1}}^{t_{k}} f_{*}(s) d_{q_{k-1}} s+\sum_{0<t_{k}<t} I_{k}\left(x_{*}\left(t_{k}\right)\right)+\int_{t_{k}}^{t} f_{*}(s) d_{q_{k}} s,
$$

for some $f_{*} \in S_{F, x_{*}}$.

Finally, we show there exists an open set $U \subseteq C(J, \mathbb{R})$ with $x \notin \mathcal{H}(x)$ for any $\lambda \in(0,1)$ and all $x \in \partial U$. Let $\lambda \in(0,1)$ and $x \in \lambda \mathcal{H}(x)$. Then there exists $v \in L^{1}(J, \mathbb{R})$ with $f \in S_{F, x}$ such that, for $t \in J$, we have

$$
x(t)=x_{0}+\sum_{0<t_{k}<t} \int_{t_{k-1}}^{t_{k}} f(s) d_{q_{k-1}} s+\sum_{0<t_{k}<t} I_{k}\left(x\left(t_{k}\right)\right)+\int_{t_{k}}^{t} f(s) d_{q_{k}} s .
$$

Repeating the computations of the second step, we have

$$
|x(t)| \leq\left|x_{0}\right|+T \psi(\|x\|)\|p\|+\sum_{k=1}^{m} c_{k}
$$

Consequently, we have

$$
\frac{\|x\|}{\left|x_{0}\right|+T \psi(\|x\|)\|p\|+\sum_{k=1}^{m} c_{k}} \leq 1 .
$$

In view of $\left(\mathrm{H}_{4}\right)$, there exists $M$ such that $\|x\| \neq M$. Let us set

$$
U=\{x \in P C(J, \mathbb{R}):\|x\|<M\} .
$$

Note that the operator $\mathcal{H}: \bar{U} \rightarrow \mathcal{P}(P C(J, \mathbb{R}))$ is upper semicontinuous and completely continuous. From the choice of $U$, there is no $x \in \partial U$ such that $x \in \lambda \mathcal{H}(x)$ for some $\lambda \in(0,1)$. Consequently, by the nonlinear alternative of Leray-Schauder type (Lemma 2.2), we deduce that $\mathcal{H}$ has a fixed point $x \in \bar{U}$ which is a solution of the problem (1.4). This completes the proof.

Example 3.4 Let us consider the following first-order initial value problem for impulsive $q_{k}$-difference inclusions:

$$
\begin{aligned}
& D_{\frac{1}{2+k}} x(t) \in F(t, x(t)), \quad t \in J=[0,1], t \neq t_{k}=\frac{k}{10}, \\
& \Delta x\left(t_{k}\right)=\frac{\left|x\left(t_{k}\right)\right|}{12+\left|x\left(t_{k}\right)\right|}, \quad k=1,2, \ldots, 9, \\
& x(0)=0 .
\end{aligned}
$$

Here $q_{k}=1 /(2+k), k=0,1,2, \ldots, 9, m=9, T=1$, and $I_{k}(x)=|x| /(12+|x|)$. We find that $\left|I_{k}(x)-I_{k}(y)\right| \leq(1 / 12)|x-y|$ and $\left|I_{k}(x)\right| \leq 1$.

(a) Let $F:[0,1] \times \mathbb{R} \rightarrow \mathcal{P}(\mathbb{R})$ be a multivalued map given by

$$
x \rightarrow F(t, x)=\left[\frac{|x|}{|x|+\sin ^{2} x+1}+t+1, e^{-x^{2}}+\frac{4}{5} t^{2}+3\right] .
$$


For $f \in F$, we have

$$
|f| \leq \max \left(\frac{|x|}{|x|+\sin ^{2} x+1}+t+1, e^{-x^{2}}+t^{2}+3\right) \leq 5, \quad x \in \mathbb{R} .
$$

Thus,

$$
\|F(t, x)\|_{\mathcal{P}}:=\sup \{|y|: y \in F(t, x)\} \leq 5=p(t) \psi(\|x\|), \quad x \in \mathbb{R},
$$

with $p(t)=1, \psi(\|x\|)=5$. Further, using the condition $\left(\mathrm{H}_{4}\right)$ we find that $M>14$. Therefore, all the conditions of Theorem 3.3 are satisfied. So, problem (3.3) with $F(t, x)$ given by (3.4) has at least one solution on $[0,1]$.

(b) If $F:[0,1] \times \mathbb{R} \rightarrow \mathcal{P}(\mathbb{R})$ is a multivalued map given by

$$
x \rightarrow F(t, x)=\left[\frac{(t+1) x^{2}}{x^{2}+1}, \frac{t|x|\left(\cos ^{2} x+1\right)}{2(|x|+1)}\right] .
$$

For $f \in F$, we have

$$
|f| \leq \max \left(\frac{(t+1) x^{2}}{x^{2}+1}, \frac{t|x|\left(\cos ^{2} x+1\right)}{2(|x|+1)}\right) \leq t+1, \quad x \in \mathbb{R} .
$$

Here $\|F(t, x)\|_{\mathcal{P}}:=\sup \{|y|: y \in F(t, x)\} \leq(t+1)=p(t) \psi(\|x\|), x \in \mathbb{R}$, with $p(t)=t+1$, $\psi(\|x\|)=1$. It is easy to verify that $M>10.5$. Then, by Theorem 3.3, the problem (3.3) with $F(t, x)$ given by $(3.5)$ has at least one solution on $[0,1]$.

\section{Second-order impulsive $q_{k}$-difference inclusions}

In this section, we study the existence of solutions for the second-order impulsive $q_{k}$ difference inclusion (1.5).

We recall the following lemma from [1].

Lemma 4.1 If $y \in C(J, \mathbb{R})$, then for any $t \in J$, the solution of the problem

$$
\begin{aligned}
& D_{q_{k}}^{2} x(t)=y(t), \quad t \in J, t \neq t_{k}, \\
& \Delta x\left(t_{k}\right)=I_{k}\left(x\left(t_{k}\right)\right), \quad k=1,2, \ldots, m, \\
& D_{q_{k}} x\left(t_{k}^{+}\right)-D_{q_{k-1}} x\left(t_{k}\right)=I_{k}^{*}\left(x\left(t_{k}\right)\right), \quad k=1,2, \ldots, m, \\
& x(0)=\alpha, \quad D_{q_{0}} x(0)=\beta,
\end{aligned}
$$

is given by

$$
\begin{aligned}
x(t)=\alpha & +\beta t \\
& +\sum_{0<t_{k}<t}\left(\int_{t_{k-1}}^{t_{k}}\left(t_{k}-q_{k-1} s-\left(1-q_{k-1}\right) t_{k-1}\right) y(s) d_{q_{k-1}} s+I_{k}\left(x\left(t_{k}\right)\right)\right) \\
& +t\left[\sum_{0<t_{k}<t}\left(\int_{t_{k-1}}^{t_{k}} f y(s) d_{q_{k-1}} s+I_{k}^{*}\left(x\left(t_{k}\right)\right)\right)\right]
\end{aligned}
$$




$$
\begin{aligned}
& -\sum_{0<t_{k}<t} t_{k}\left(\int_{t_{k-1}}^{t_{k}} y(s) d_{q_{k-1}} s+I_{k}^{*}\left(x\left(t_{k}\right)\right)\right) \\
& +\int_{t_{k}}^{t}\left(t-q_{k} s-\left(1-q_{k}\right) t_{k}\right) y(s) d_{q_{k}} s
\end{aligned}
$$

with $\sum_{0<0}(\cdot)=0$.

Definition 4.2 A function $x \in P C(J, \mathbb{R})$ is said to be a solution of $(1.5)$ if $x(0)=x_{0}$, $D_{q_{0}} x(0)=\beta, \Delta x\left(t_{k}\right)=I_{k}\left(x\left(t_{k}\right)\right), D_{q_{k}} x\left(t_{k}^{+}\right)-D_{q_{k-1}} x\left(t_{k}\right)=I_{k}^{*}\left(x\left(t_{k}\right)\right), k=1,2, \ldots, m$ and there exists $f \in L^{1}(J, \mathbb{R})$ such that $f(t) \in F(t, x(t))$ on $J$ and

$$
\begin{aligned}
x(t)=\alpha & +\beta t \\
& +\sum_{0<t_{k}<t}\left(\int_{t_{k-1}}^{t_{k}}\left(t_{k}-q_{k-1} s-\left(1-q_{k-1}\right) t_{k-1}\right) f(s) d_{q_{k-1}} s+I_{k}\left(x\left(t_{k}\right)\right)\right) \\
& +t\left[\sum_{0<t_{k}<t}\left(\int_{t_{k-1}}^{t_{k}} f(s) d_{q_{k-1}} s+I_{k}^{*}\left(x\left(t_{k}\right)\right)\right)\right] \\
& -\sum_{0<t_{k}<t} t_{k}\left(\int_{t_{k-1}}^{t_{k}} f(s) d_{q_{k-1}} s+I_{k}^{*}\left(x\left(t_{k}\right)\right)\right) \\
& +\int_{t_{k}}^{t}\left(t-q_{k} s-\left(1-q_{k}\right) t_{k}\right) f(s) d_{q_{k}} s
\end{aligned}
$$

with $\sum_{0<0}(\cdot)=0$.

Theorem 4.3 Assume that $\left(\mathrm{H}_{1}\right),\left(\mathrm{H}_{2}\right)$ hold. In addition we suppose that:

$\left(\mathrm{A}_{1}\right)$ there exist constants $c_{k}, c_{k}^{*}$ such that $\left|I_{k}(x)\right| \leq c_{k},\left|I_{k}^{*}(y)\right| \leq c_{k}^{*}, k=1,2, \ldots$, m for each $x, y \in \mathbb{R}$

$\left(\mathrm{A}_{2}\right)$ there exists a constant $M>0$ such that

$$
\frac{M}{|\alpha|+|\beta| T+\|p\| \psi(M) \Lambda_{1}+\sum_{k=1}^{m}\left[c_{k}+c_{k}^{*}\left(T+t_{k}\right)\right]}>1
$$

where

$$
\Lambda_{1}=\sum_{k=1}^{m+1} \frac{\left(t_{k}-t_{k-1}\right)^{2}}{1+q_{k-1}}+\sum_{k=1}^{m}\left(T+t_{k}\right)\left(t_{k}-t_{k-1}\right)
$$

Then the initial value problem (1.5) has at least one solution on J.

Proof Define the operator $\mathcal{H}: P C(J, \mathbb{R}) \rightarrow \mathcal{P}(P C(J, \mathbb{R}))$ by

$$
\begin{aligned}
\mathcal{H}(x)=h \in P C(J, \mathbb{R}): h(t)= & \alpha+\beta t+\sum_{0<t_{k}<t}\left(\int_{t_{k-1}}^{t_{k}}\left(t_{k}-q_{k-1} s-\left(1-q_{k-1}\right) t_{k-1}\right) f(s) d_{q_{k-1}} s\right. \\
& \left.+I_{k}\left(x\left(t_{k}\right)\right)\right)+t\left[\sum_{0<t_{k}<t}\left(\int_{t_{k-1}}^{t_{k}} f(s) d_{q_{k-1}} s+I_{k}^{*}\left(x\left(t_{k}\right)\right)\right)\right]
\end{aligned}
$$




$$
\begin{aligned}
& -\sum_{0<t_{k}<t} t_{k}\left(\int_{t_{k-1}}^{t_{k}} f(s) d_{q_{k-1}} s+I_{k}^{*}\left(x\left(t_{k}\right)\right)\right) \\
& +\int_{t_{k}}^{t}\left(t-q_{k} s-\left(1-q_{k}\right) t_{k}\right) f(s) d_{q_{k}} s,
\end{aligned}
$$

for $f \in S_{F, x}$.

We will show that $\mathcal{H}$ satisfies the assumptions of the nonlinear alternative of LeraySchauder type. The proof consists of several steps. As a first step, we show that $\mathcal{H}$ is convex for each $x \in P C(J, \mathbb{R})$. This step is obvious since $S_{F, x}$ is convex ( $F$ has convex values), and therefore we omit the proof.

In the second step, we show that $\mathcal{H}$ maps bounded sets (balls) into bounded sets in $P C(J, \mathbb{R})$. For a positive number $\rho$, let $B_{\rho}=\{x \in P C(J, \mathbb{R}):\|x\| \leq \rho\}$ be a bounded ball in $P C(J, \mathbb{R})$. Then, for each $h \in \mathcal{H}(x), x \in B_{\rho}$, there exists $f \in S_{F, x}$ such that

$$
\begin{aligned}
h(t)=\alpha & +\beta t \\
& +\sum_{0<t_{k}<t}\left(\int_{t_{k-1}}^{t_{k}}\left(t_{k}-q_{k-1} s-\left(1-q_{k-1}\right) t_{k-1}\right) f(s) d_{q_{k-1}} s+I_{k}\left(x\left(t_{k}\right)\right)\right) \\
& +t\left[\sum_{0<t_{k}<t}\left(\int_{t_{k-1}}^{t_{k}} f(s) d_{q_{k-1}} s+I_{k}^{*}\left(x\left(t_{k}\right)\right)\right)\right] \\
& -\sum_{0<t_{k}<t} t_{k}\left(\int_{t_{k-1}}^{t_{k}} f(s) d_{q_{k-1}} s+I_{k}^{*}\left(x\left(t_{k}\right)\right)\right) \\
& +\int_{t_{k}}^{t}\left(t-q_{k} s-\left(1-q_{k}\right) t_{k}\right) f(s) d_{q_{k}} s .
\end{aligned}
$$

Then for $t \in J$ we have

$$
\begin{aligned}
|h(t)| \leq & |\alpha|+|\beta| t \\
& +\sum_{0<t_{k}<t}\left(\int_{t_{k-1}}^{t_{k}}\left(t_{k}-q_{k-1} s-\left(1-q_{k-1}\right) t_{k-1}\right)|f(s)| d_{q_{k-1}} s+\left|I_{k}\left(x\left(t_{k}\right)\right)\right|\right) \\
& +t\left[\sum_{0<t_{k}<t}\left(\int_{t_{k-1}}^{t_{k}}|f(s)| d_{q_{k-1}} s+\left|I_{k}^{*}\left(x\left(t_{k}\right)\right)\right|\right)\right] \\
& +\sum_{0<t_{k}<t} t_{k}\left(\int_{t_{k-1}}^{t_{k}}|f(s)| d_{q_{k-1}} s+\left|I_{k}^{*}\left(x\left(t_{k}\right)\right)\right|\right) \\
& +\int_{t_{k}}^{t}\left(t-q_{k} s-\left(1-q_{k}\right) t_{k}\right)|f(s)| d_{q_{k}} s \\
\leq & |\alpha|+|\beta| T \\
& +\sum_{0<t_{k}<T}\left(\int_{t_{k-1}}^{t_{k}}\left(t_{k}-q_{k-1} s-\left(1-q_{k-1}\right) t_{k-1}\right) p(s) \psi(\|x\|) d_{q_{k-1}} s+\left|I_{k}\left(x\left(t_{k}\right)\right)\right|\right) \\
& +T\left[\sum_{0<t_{k}<T}\left(\int_{t_{k-1}}^{t_{k}} p(s) \psi(\|x\|) d_{q_{k-1}} s+\left|I_{k}^{*}\left(x\left(t_{k}\right)\right)\right|\right)\right] \\
& +\sum_{0<t_{k}<T} t_{k}\left(\int_{t_{k-1}}^{t_{k}} p(s) \psi(\|x\|) d_{q_{k-1}} s+\left|I_{k}^{*}\left(x\left(t_{k}\right)\right)\right|\right)
\end{aligned}
$$




$$
\begin{aligned}
& +\int_{t_{m}}^{T}\left(T-q_{m} s-\left(1-q_{m}\right) t_{m}\right) p(s) \psi(\|x\|) d_{q_{m}} s \\
= & |\alpha|+|\beta| T+\sum_{k=1}^{m}\left(\frac{\left(t_{k}-t_{k-1}\right)^{2}}{1+q_{k-1}}\|p\| \psi(\|x\|)+c_{k}\right) \\
& +T\left[\sum_{k=1}^{m}\left(\|p\| \psi(\|x\|)\left(t_{k}-t_{k-1}\right)+c_{k}^{*}\right)\right] \\
& +\sum_{k=1}^{m} t_{k}\left(\|p\| \psi(\|x\|)\left(t_{k}-t_{k-1}\right)+c_{k}^{*}\right)+\frac{\left(T-t_{m}\right)^{2}}{1+q_{m}}\|p\| \psi(\|x\|) \\
= & |\alpha|+|\beta| T+\|p\| \psi(\|x\|)\left\{\sum_{k=1}^{m+1} \frac{\left(t_{k}-t_{k-1}\right)^{2}}{1+q_{k-1}}+\sum_{k=1}^{m}\left(T+t_{k}\right)\left(t_{k}-t_{k-1}\right)\right\} \\
& +\sum_{k=1}^{m}\left[c_{k}+c_{k}^{*}\left(T+t_{k}\right)\right] .
\end{aligned}
$$

Consequently,

$$
\begin{aligned}
\|h\| \leq & |\alpha|+|\beta| T+\|p\| \psi(\rho)\left\{\sum_{k=1}^{m+1} \frac{\left(t_{k}-t_{k-1}\right)^{2}}{1+q_{k-1}}+\sum_{k=1}^{m}\left(T+t_{k}\right)\left(t_{k}-t_{k-1}\right)\right\} \\
& +\sum_{k=1}^{m}\left[c_{k}+c_{k}^{*}\left(T+t_{k}\right)\right] .
\end{aligned}
$$

Now we show that $\mathcal{H}$ maps bounded sets into equicontinuous sets of $P C(J, \mathbb{R})$. Let $\tau_{1}, \tau_{2} \in J, \tau_{1}<\tau_{2}$ with $\tau_{1} \in J_{u}, \tau_{2} \in J_{v}, u \leq v$ for some $u, v \in\{0,1,2, \ldots, m\}$ and $x \in B_{\rho}$. For each $h \in \mathcal{H}(x)$, we obtain

$$
\begin{aligned}
\left|h\left(\tau_{2}\right)-h\left(\tau_{1}\right)\right| \leq & |\beta|\left|\tau_{2}-\tau_{1}\right| \\
& +\sum_{\tau_{1}<t_{k}<\tau_{2}}\left(\int_{t_{k-1}}^{t_{k}}\left(t_{k}-q_{k-1} s-\left(1-q_{k-1}\right) t_{k-1}\right)|f(s)| d_{q_{k-1}} s+\left|I_{k}\left(x\left(t_{k}\right)\right)\right|\right) \\
& +\left|\tau_{2}-\tau_{1}\right|\left[\sum_{0<t_{k}<\tau_{1}}\left(\int_{t_{k-1}}^{t_{k}}|f(s)| d_{q_{k-1}} s+\left|I_{k}^{*}\left(x\left(t_{k}\right)\right)\right|\right)\right] \\
& +\tau_{2}\left[\sum_{\tau_{1}<t_{k}<\tau_{2}}\left(\int_{t_{k-1}}^{t_{k}}|f(s)| d_{q_{k-1}} s+\left|I_{k}^{*}\left(x\left(t_{k}\right)\right)\right|\right)\right] \\
& +\sum_{\tau_{1}<t_{k}<\tau_{2}} t_{k}\left(\int_{t_{k-1}}^{t_{k}}|f(s)| d_{q_{k-1}} s+\left|I_{k}^{*}\left(x\left(t_{k}\right)\right)\right|\right) \\
& +\left|\int_{t_{v}}^{\tau_{2}}\left(\tau_{2}-q_{k} s-\left(1-q_{k}\right) t_{k}\right)\right| f(s) \mid d_{q_{k}} s \\
& -\int_{t_{u}}^{\tau_{1}}\left(\tau_{1}-q_{k} s-\left(1-q_{k}\right) t_{k}\right)|f(s)| d_{q_{k}} s \mid .
\end{aligned}
$$

Obviously the right hand side of the above inequality tends to zero independently of $x \in$ $B_{\rho}$ as $\tau_{2}-\tau_{1} \rightarrow 0$. Therefore it follows by the Arzelá-Ascoli theorem that $\mathcal{H}: P C(J, \mathbb{R}) \rightarrow$ $\mathcal{P}(P C(J, \mathbb{R}))$ is completely continuous. 
Since $\mathcal{H}$ is completely continuous, in order to prove that it is upper semicontinuous it is enough to prove that it has a closed graph. Thus, in our next step, we show that $\mathcal{H}$ has $a$ closed graph. Let $x_{n} \rightarrow x_{*}, h_{n} \in \mathcal{H}\left(x_{n}\right)$ and $h_{n} \rightarrow h_{*}$. Then we need to show that $h_{*} \in \mathcal{H}\left(x_{*}\right)$. Associated with $h_{n} \in \mathcal{H}\left(x_{n}\right)$, there exists $f_{n} \in S_{F, x_{n}}$ such that, for each $t \in J$,

$$
\begin{aligned}
h_{n}(t)= & \alpha+\beta t+\sum_{0<t_{k}<t}\left(\int_{t_{k-1}}^{t_{k}}\left(t_{k}-q_{k-1} s-\left(1-q_{k-1}\right) t_{k-1}\right) f_{n}(s) d_{q_{k-1}} s+I_{k}\left(x\left(t_{k}\right)\right)\right) \\
& +t\left[\sum_{0<t_{k}<t}\left(\int_{t_{k-1}}^{t_{k}} f_{n}(s) d_{q_{k-1}} s+I_{k}^{*}\left(x\left(t_{k}\right)\right)\right)\right] \\
& -\sum_{0<t_{k}<t} t_{k}\left(\int_{t_{k-1}}^{t_{k}} f_{n}(s) d_{q_{k-1}} s+I_{k}^{*}\left(x\left(t_{k}\right)\right)\right) \\
& +\int_{t_{k}}^{t}\left(t-q_{k} s-\left(1-q_{k}\right) t_{k}\right) f_{n}(s) d_{q_{k}} s .
\end{aligned}
$$

Thus it suffices to show that there exists $f_{*} \in S_{F, x_{*}}$ such that, for each $t \in J$,

$$
\begin{aligned}
h_{*}(t)= & \alpha+\beta t+\sum_{0<t_{k}<t}\left(\int_{t_{k-1}}^{t_{k}}\left(t_{k}-q_{k-1} s-\left(1-q_{k-1}\right) t_{k-1}\right) f_{*}(s) d_{q_{k-1}} s+I_{k}\left(x\left(t_{k}\right)\right)\right) \\
& +t\left[\sum_{0<t_{k}<t}\left(\int_{t_{k-1}}^{t_{k}} f_{*}(s) d_{q_{k-1}} s+I_{k}^{*}\left(x\left(t_{k}\right)\right)\right)\right] \\
& -\sum_{0<t_{k}<t} t_{k}\left(\int_{t_{k-1}}^{t_{k}} f_{*}(s) d_{q_{k-1}} s+I_{k}^{*}\left(x\left(t_{k}\right)\right)\right) \\
& +\int_{t_{k}}^{t}\left(t-q_{k} s-\left(1-q_{k}\right) t_{k}\right) f_{*}(s) d_{q_{k}} s .
\end{aligned}
$$

Let us consider the linear operator $\Theta: L^{1}(J, \mathbb{R}) \rightarrow P C(J, \mathbb{R})$ given by

$$
\begin{aligned}
f \mapsto \Theta(f)(t)= & \alpha+\beta t+\sum_{0<t_{k}<t}\left(\int_{t_{k-1}}^{t_{k}}\left(t_{k}-q_{k-1} s-\left(1-q_{k-1}\right) t_{k-1}\right) f(s) d_{q_{k-1}} s+I_{k}\left(x\left(t_{k}\right)\right)\right) \\
& +t\left[\sum_{0<t_{k}<t}\left(\int_{t_{k-1}}^{t_{k}} f(s) d_{q_{k-1}} s+I_{k}^{*}\left(x\left(t_{k}\right)\right)\right)\right] \\
& -\sum_{0<t_{k}<t} t_{k}\left(\int_{t_{k-1}}^{t_{k}} f(s) d_{q_{k-1}} s+I_{k}^{*}\left(x\left(t_{k}\right)\right)\right) \\
& +\int_{t_{k}}^{t}\left(t-q_{k} s-\left(1-q_{k}\right) t_{k}\right) f(s) d_{q_{k}} s .
\end{aligned}
$$

Observe that

$$
\begin{aligned}
\left\|h_{n}(t)-h_{*}(t)\right\|= & \| \sum_{0<t_{k}<t} \int_{t_{k-1}}^{t_{k}}\left(t_{k}-q_{k-1} s-\left(1-q_{k-1}\right) t_{k-1}\right)\left(f_{n}(u)-f_{*}(u)\right) d_{q_{k-1}} s \\
& +\sum_{0<t_{k}<t}\left|I_{k}\left(x_{n}\left(t_{k}\right)\right)-I_{k}\left(x_{*}\left(t_{k}\right)\right)\right|
\end{aligned}
$$




$$
\begin{aligned}
& +T \sum_{0<t_{k}<t} \int_{t_{k-1}}^{t_{k}}\left(f_{n}(u)-f_{*}(u)\right) d_{q_{k-1}} s \\
& +T \sum_{0<t_{k}<t}\left|I_{k}^{*}\left(x_{n}\left(t_{k}\right)\right)-I_{k}^{*}\left(x_{*}\left(t_{k}\right)\right)\right| \\
& +\sum_{0<t_{k}<t} t_{k} \int_{t_{k-1}}^{t_{k}}\left(f_{n}(u)-f_{*}(u)\right) d_{q_{k-1}} s \\
& +\sum_{0<t_{k}<t}\left|I_{k}^{*}\left(x_{n}\left(t_{k}\right)\right)-I_{k}^{*}\left(x_{*}\left(t_{k}\right)\right)\right| \\
& +\int_{t_{k}}^{t}\left(t-q_{k} s-\left(1-q_{k}\right) t_{k}\right)\left(f_{n}(u)-f_{*}(u)\right) d_{q_{k}} s \| \rightarrow 0,
\end{aligned}
$$

as $n \rightarrow \infty$.

Thus, it follows by Lemma 2.3 that $\Theta \circ S_{F}$ is a closed graph operator. Further, we have $h_{n}(t) \in \Theta\left(S_{F, x_{n}}\right)$. Since $x_{n} \rightarrow x_{*}$, therefore, we have

$$
\begin{aligned}
h_{*}(t)= & \alpha+\beta t+\sum_{0<t_{k}<t}\left(\int_{t_{k-1}}^{t_{k}}\left(t_{k}-q_{k-1} s-\left(1-q_{k-1}\right) t_{k-1}\right) f_{*}(s) d_{q_{k-1}} s+I_{k}\left(x\left(t_{k}\right)\right)\right) \\
& +t\left[\sum_{0<t_{k}<t}\left(\int_{t_{k-1}}^{t_{k}} f_{*}(s) d_{q_{k-1}} s+I_{k}^{*}\left(x\left(t_{k}\right)\right)\right)\right] \\
& -\sum_{0<t_{k}<t} t_{k}\left(\int_{t_{k-1}}^{t_{k}} f_{*}(s) d_{q_{k-1}} s+I_{k}^{*}\left(x\left(t_{k}\right)\right)\right) \\
& +\int_{t_{k}}^{t}\left(t-q_{k} s-\left(1-q_{k}\right) t_{k}\right) f_{*}(s) d_{q_{k}} s,
\end{aligned}
$$

for some $f_{*} \in S_{F, x_{*}}$.

Finally, we show there exists an open set $U \subseteq C(J, \mathbb{R})$ with $x \notin \mathcal{H}(x)$ for any $\lambda \in(0,1)$ and all $x \in \partial U$. Let $\lambda \in(0,1)$ and $x \in \lambda \mathcal{H}(x)$. Then there exists $f \in L^{1}(J, \mathbb{R})$ with $f \in S_{F, x}$ such that, for $t \in J$, we have

$$
\begin{aligned}
x(t)= & \alpha+\beta t+\sum_{0<t_{k}<t}\left(\int_{t_{k-1}}^{t_{k}}\left(t_{k}-q_{k-1} s-\left(1-q_{k-1}\right) t_{k-1}\right) f(s) d_{q_{k-1}} s+I_{k}\left(x\left(t_{k}\right)\right)\right) \\
& +t\left[\sum_{0<t_{k}<t}\left(\int_{t_{k-1}}^{t_{k}} f(s) d_{q_{k-1}} s+I_{k}^{*}\left(x\left(t_{k}\right)\right)\right)\right] \\
& -\sum_{0<t_{k}<t} t_{k}\left(\int_{t_{k-1}}^{t_{k}} f(s) d_{q_{k-1}} s+I_{k}^{*}\left(x\left(t_{k}\right)\right)\right) \\
& +\int_{t_{k}}^{t}\left(t-q_{k} s-\left(1-q_{k}\right) t_{k}\right) f(s) d_{q_{k}} s .
\end{aligned}
$$

Repeating the computations of the second step, we have

$$
\begin{aligned}
|x(t)| \leq & |\alpha|+|\beta| T+\|p\| \psi(\|x\|)\left\{\sum_{k=1}^{m+1} \frac{\left(t_{k}-t_{k-1}\right)^{2}}{1+q_{k-1}}+\sum_{k=1}^{m}\left(T+t_{k}\right)\left(t_{k}-t_{k-1}\right)\right\} \\
& +\sum_{k=1}^{m}\left[c_{k}+c_{k}^{*}\left(T+t_{k}\right)\right] .
\end{aligned}
$$


Consequently, we have

$$
\frac{\|x\|}{|\alpha|+|\beta| T+\|p\| \psi(\|x\|) \Lambda_{1}+\sum_{k=1}^{m}\left[c_{k}+c_{k}^{*}\left(T+t_{k}\right)\right]} \leq 1 .
$$

In view of $\left(\mathrm{A}_{2}\right)$, there exists $M$ such that $\|x\| \neq M$. Let us set

$$
U=\{x \in P C(J, \mathbb{R}):\|x\|<M\} .
$$

Note that the operator $\mathcal{H}: \bar{U} \rightarrow \mathcal{P}(P C(J, \mathbb{R}))$ is upper semicontinuous and completely continuous. From the choice of $U$, there is no $x \in \partial U$ such that $x \in \lambda \mathcal{H}(x)$ for some $\lambda \in(0,1)$. Consequently, by the nonlinear alternative of Leray-Schauder type (Lemma 2.2), we deduce that $\mathcal{H}$ has a fixed point $x \in \bar{U}$ which is a solution of the problem (1.4). This completes the proof.

Example 4.4 Let us consider the following second-order impulsive $q_{k}$-difference inclusion with initial conditions:

$$
\left\{\begin{array}{l}
D_{\frac{2}{3+k}}^{2} x(t) \in F(t, x(t)), \quad t \in J=[0,1], t \neq t_{k}=\frac{k}{10}, \\
\Delta x\left(t_{k}\right)=\frac{\left|x\left(t_{k}\right)\right|}{15\left(6+\left|x\left(t_{k}\right)\right|\right)}, \quad k=1,2, \ldots, 9, \\
D_{\frac{2}{3+k}} x\left(t_{k}^{+}\right)-D_{\frac{2}{3+k-1}} x\left(t_{k}\right)=\frac{\left|x\left(t_{k}\right)\right|}{19\left(3+\left|x\left(t_{k}\right)\right|\right)}, \quad k=1,2, \ldots, 9, \\
x(0)=0, \quad D_{\frac{2}{3}} x(0)=0 .
\end{array}\right.
$$

Here $q_{k}=2 /(3+k), k=0,1,2, \ldots, 9, m=9, T=1, \alpha=0, \beta=0, I_{k}(x)=|x| /(15(6+|x|))$, and $I_{k}^{*}(x)=|x| /(19(3+|x|))$. We find that $\left|I_{k}(x)-I_{k}(y)\right| \leq(1 / 90)|x-y|,\left|I_{k}^{*}(x)-I_{k}^{*}(y)\right| \leq$ $(1 / 57)|x-y|$, and $I_{k}(x) \leq 1 / 15, I_{k}^{*}(x) \leq 1 / 19$; and we have

$$
\Lambda_{1}=\sum_{k=1}^{m+1} \frac{\left(t_{k}-t_{k-1}\right)^{2}}{1+q_{k-1}}+\sum_{k=1}^{m}\left(T+t_{k}\right)\left(t_{k}-t_{k-1}\right) \approx 1.42663542 .
$$

(a) Let $F:[0,1] \times \mathbb{R} \rightarrow \mathcal{P}(\mathbb{R})$ be a multivalued map given by

$$
x \rightarrow F(t, x)=\left[\frac{|x|}{|x|+\sin ^{2} x+1}+t+1, e^{-x^{2}}+\frac{4}{5} t^{2}+3\right] .
$$

For $f \in F$, we have

$$
|f| \leq \max \left(\frac{|x|}{|x|+\sin ^{2} x+1}+t+1, e^{-x^{2}}+t^{2}+3\right) \leq 5, \quad x \in \mathbb{R} .
$$

Thus,

$$
\|F(t, x)\|_{\mathcal{P}}:=\sup \{|y|: y \in F(t, x)\} \leq 5=p(t) \psi(\|x\|), \quad x \in \mathbb{R},
$$

with $p(t)=1, \psi(\|x\|)=5$. Further, using the condition $\left(\mathrm{A}_{2}\right)$ we find

$$
\frac{M}{5 \Lambda_{1}+\sum_{k=1}^{9}\left[\frac{1}{15}+\frac{1}{19}\left(1+t_{k}\right)\right]}>1,
$$


which implies $M>8.44370316$. Therefore, all the conditions of Theorem 4.3 are satisfied. So, problem (4.5) with $F(t, x)$ given by $(4.6)$ has at least one solution on $[0,1]$.

(b) If $F:[0,1] \times \mathbb{R} \rightarrow \mathcal{P}(\mathbb{R})$ is a multivalued map given by

$$
x \rightarrow F(t, x)=\left[\frac{(t+1) x^{2}}{x^{2}+1}, \frac{t|x|\left(\cos ^{2} x+1\right)}{2(|x|+1)}\right] .
$$

For $f \in F$, we have

$$
|f| \leq \max \left(\frac{(t+1) x^{2}}{x^{2}+1}, \frac{t|x|\left(\cos ^{2} x+1\right)}{2(|x|+1)}\right) \leq t+1, \quad x \in \mathbb{R}
$$

Here $\|F(t, x)\|_{\mathcal{P}}:=\sup \{|y|: y \in F(t, x)\} \leq(t+1)=p(t) \psi(\|x\|), x \in \mathbb{R}$, with $p(t)=t+1$, $\psi(\|x\|)=1$. It is easy to verify that $M>3.45047945$. Then, by Theorem 4.3 , the problem (4.5) with $F(t, x)$ given by (4.7) has at least one solution on $[0,1]$.

\section{Competing interests}

The authors declare that they have no competing interests.

\section{Authors' contributions}

All authors contributed equally to this article. They read and approved the final manuscript.

\section{Author details}

${ }^{1}$ Department of Mathematics, University of loannina, loannina, 451 10, Greece. ${ }^{2}$ Department of Mathematics, Faculty of Applied Science, Nonlinear Dynamic Analysis Research Center, King Mongkut's University of Technology North Bangkok, Bangkok, 10800, Thailand. ${ }^{3}$ Centre of Excellence in Mathematics, CHE, Si Ayutthaya Rd., Bangkok, 10400, Thailand.

\section{Authors' information}

Sotiris K Ntouyas is a member of Nonlinear Analysis and Applied Mathematics (NAAM)-Research Group at King Abdulaziz University, Jeddah, Saudi Arabia.

\section{Acknowledgements}

This research is supported by the Centre of Excellence in Mathematics, the Commission on Higher Education, Thailand.

Received: 16 June 2014 Accepted: 30 September 2014 Published: 13 Oct 2014

\section{References}

1. Tariboon, J, Ntouyas, SK: Quantum calculus on finite intervals and applications to impulsive difference equations. Adv. Differ. Equ. 2013, Article ID 282 (2013)

2. Kac, V, Cheung, P: Quantum Calculus. Springer, New York (2002)

3. Bangerezako, G: Variational q-calculus. J. Math. Anal. Appl. 289, 650-665 (2004)

4. Dobrogowska, A, Odzijewicz, A: Second order $q$-difference equations solvable by factorization method. J. Comput. Appl. Math. 193, 319-346 (2006)

5. Gasper, G, Rahman, M: Some systems of multivariable orthogonal q-Racah polynomials. Ramanujan J. 13, 389-405 (2007)

6. Ismail, MEH, Simeonov, P: q-Difference operators for orthogonal polynomials. J. Comput. Appl. Math. 233, 749-761 (2009)

7. Bohner, M, Guseinov, GS: The $h$-Laplace and q-Laplace transforms. J. Math. Anal. Appl. 365, 75-92 (2010)

8. El-Shahed, M, Hassan, HA: Positive solutions of q-difference equation. Proc. Am. Math. Soc. 138, 1733-1738 (2010)

9. Ahmad, B: Boundary-value problems for nonlinear third-order q-difference equations. Electron. J. Differ. Equ. 2011, 94 (2011)

10. Ahmad, B, Alsaedi, A, Ntouyas, SK: A study of second-order q-difference equations with boundary conditions. Adv. Differ. Equ. 2012, Article ID 35 (2012)

11. Ahmad, B, Ntouyas, SK, Purnaras, IK: Existence results for nonlinear q-difference equations with nonlocal boundary conditions. Commun. Appl. Nonlinear Anal. 19, 59-72 (2012)

12. Ahmad, B, Nieto, Jj: On nonlocal boundary value problems of nonlinear q-difference equations. Adv. Differ. Equ. 2012, Article ID 81 (2012)

13. Ahmad, B, Ntouyas, SK: Boundary value problems for q-difference inclusions. Abstr. Appl. Anal. 2011, Article ID 292860 (2011)

14. Zhou, W, Liu, H: Existence solutions for boundary value problem of nonlinear fractional q-difference equations. Adv. Differ. Equ. 2013, Article ID 113 (2013)

15. Yu, C, Wang, J: Existence of solutions for nonlinear second-order $q$-difference equations with first-order q-derivatives Adv. Differ. Equ. 2013, Article ID 124 (2013)

16. Lakshmikantham, V, Bainov, DD, Simeonov, PS: Theory of Impulsive Differential Equations. World Scientific, Singapore (1989) 
17. Samoilenko, AM, Perestyuk, NA: Impulsive Differential Equations. World Scientific, Singapore (1995)

18. Benchohra, M, Henderson, J, Ntouyas, SK: Impulsive Differential Equations and Inclusions, vol. 2. Hindawi Publishing Corporation, New York (2006)

19. Bohner, M, Peterson, A: Dynamic Equations on Time Scales. An Introduction with Applications. Birkhäuser Boston, Boston (2001)

20. Deimling, K: Multivalued Differential Equations. de Gruyter, Berlin (1992)

21. Hu, S, Papageorgiou, N: Handbook of Multivalued Analysis. Vol. I. Theory. Kluwer Academic, Dordrecht (1997)

22. Granas, A, Dugundji, J: Fixed Point Theory. Springer, New York (2005)

23. Lasota, A, Opial, Z: An application of the Kakutani-Ky Fan theorem in the theory of ordinary differential equations. Bull. Acad. Pol. Sci., Sér. Sci. Math. Astron. Phys. 13, 781-786 (1965)

24. Frigon, M: Théorèmes d'existence de solutions d'inclusions différentielles. In: Granas, A, Frigon, M (eds.) Topological Methods in Differential Equations and Inclusions. NATO ASI Series C, vol. 472, pp. 51-87. Kluwer Academic, Dordrecht (1995)

10.1186/1687-1847-2014-262

Cite this article as: Ntouyas and Tariboon: Applications of quantum calculus on finite intervals to impulsive

difference inclusions. Advances in Difference Equations 2014, 2014:262

\section{Submit your manuscript to a SpringerOpen ${ }^{\circ}$ journal and benefit from:}

- Convenient online submission

- Rigorous peer review

- Immediate publication on acceptance

Open access: articles freely available online

- High visibility within the field

- Retaining the copyright to your article 